\title{
Improving the Metrics and Data Reporting for Maternal Mortality: A Challenge to Public Health Surveillance and Effective Prevention
}

\author{
James Studnicki, ScD ${ }^{1}{ }^{*}$, David C. Reardon, PhD ${ }^{2}$, Donna J. Harrison, M.D. ${ }^{3}$, John W. Fisher, \\ PhD ${ }^{1}$, Ingrid Skop, M.D. ${ }^{3}$ \\ ${ }^{1}$ Charlotte Lozier Institute, Arlington, Virginia \\ ${ }^{2}$ Elliot Institute, Springfield, Illinois \\ ${ }^{3}$ American Association of Pro-life Obstetricians and Gynecologists, Eau Claire, Michigan
}

\begin{abstract}
Background: The current measuring metric and reporting methods for assessing maternal mortality are seriously flawed. Evidence-based prevention strategies require consistently reported surveillance data and validated measurement metrics.

Main Body: The denominator of live births used in the maternal mortality ratio reinforces the mistaken notion that all maternal deaths are consequent to a live birth and, at the same time, inappropriately inflates the value of the ratio for subpopulations of women with the highest percentage of pregnancies ending in outcomes other than a live birth. Inadequate methods for identifying induced or spontaneous abortion complications assure that most maternal deaths associated with those pregnancy outcomes are unlikely to be attributed. Absent the ability to identify all maternal deaths, and without the ability to differentiate those deaths by specific pregnancy outcomes, existing variations in pregnancy outcome-specific maternal deaths are masked by the use of an aggregated (all outcome) numerator. Under these circumstances, clear and accurate data is not available to inform evidence-based preventive strategies. As the result, algorithms applied for analyzing maternal mortality data may return distorted results.

Conclusion: Improvement in the effectiveness of maternal mortality surveillance will require: mandatory certification of all fetal losses; linkage of death, birth and all fetal loss (induced and natural) certificates; modification of the structure of the overall maternal mortality ratio to enable pregnancy outcome-specific ratio calculations; development of the appropriate ICD codes which are specific to induced and spontaneous abortions; education for providers on identifying and reporting early pregnancy losses; and, flexible information systems and methods which integrate these capabilities and inform users.
\end{abstract}

Keywords: maternal mortality, maternal mortality ratio, pregnancy outcomes, induced abortion, natural fetal loss, ICD-10 coding 


\begin{abstract}
Abbreviations:
CDC: Centers for Disease Control and Prevention

ICD: International Classification of Diseases

MMR: Maternal Mortality Ratio

NHB: Non-Hispanic Black

NHW: Non-Hispanic White

TFR: Total Fertility Rate

WHO: World Health Organization

*Corresponding author: jstudnicki@lozierinstitute.org

DOI: 10.5210/ojphi.v11i2.10012

Copyright @2019 the author(s)

This is an Open Access article. Authors own copyright of their articles appearing in the Online Journal of Public Health Informatics. Readers may copy articles without permission of the copyright owner(s), as long as the author and OJPHI are acknowledged in the copy and the copy is used for educational, not-for-profit purposes.
\end{abstract}

\title{
Background
}

Maternal mortality is the death of a woman during her pregnancy or within some fixed time from the termination of her pregnancy. Definitions in use differ on the termination-to-death time frame applied (42 days, 365 days, or greater than one year) and whether the pregnancy alone is the sole criterion for a mortality relationship (World Health Organization/ WHO) or requires a proximate nexus of the pregnancy to the death (Centers for Disease Control and Prevention/CDC) [1,2]. These differing definitions, the practical difficulties involved in linking a maternal death to all pregnancy outcomes, limitations associated with the International Classification of Diseases (ICD10) coding of maternal death causes, and inconsistent and incomplete reporting have handicapped the process of maternal mortality surveillance in the U.S [3]. Additionally, fundamental structural and conceptual flaws impair maternal mortality surveillance worldwide. The denominator employed to compute comparative metrics in all of these definitions is the number of live births and not the number of pregnancies, conveying the false impression that all maternal deaths are consequent to a live birth. Pregnancy, of course, may end in many ways: a live birth, an induced abortion, or a natural fetal loss (stillbirth, ectopic pregnancy, spontaneous abortion, molar pregnancy) occurring at any time during gestation. A pregnancy that does not result in a live birth will not be included in the denominator. If the outcome of the pregnancy is spontaneous or induced abortion it is unlikely to be included in any numerator due to coding limitations and lack of mandatory reporting. Although there are over 2000 obstetric codes in ICD-10, only twelve codes cover complications of induced abortion. These codes can be difficult to locate, as most search 
mechanisms will lead to spontaneous abortion codes rather than induced abortion codes, and there are no specific codes for psychologic sequelae, transfusion, and return to operating room or death related to abortion. There are also no codes to specify whether a complication results from a medical versus a surgical abortion. In some sub-populations, non-Hispanic Blacks (NHB) in the U.S. for example, in which a substantial proportion of pregnancies do not result in a live birth, these definitional and coding limitations introduce significant distortions into "maternal mortality" statistics and, subsequently, the analytical methods applied to them.

\section{Main Text}

\section{Pregnancy Outcomes and Maternal Mortality Metrics}

In the U.S. in calendar year 2009, there were $6,369,000$ pregnancies; $64.8 \%$ resulted in live births, $18.1 \%$ in induced abortions and $17.1 \%$ in natural fetal losses [4]. Only the live birth number, representing less than two thirds of all pregnancies, may be considered reliable. The numbers of induced abortions and natural fetal losses are crude estimates based on incomplete state reports and infrequently conducted small sample surveys. This data gap is particularly problematic for the category of natural or induced fetal losses for two reasons. First, research has indicated that pregnant women who do not successfully carry to term may be at significantly greater risk of premature mortality, often from emotional or social factors such as suicide or violence whether the fetal loss was by induced abortion or a spontaneous loss [5,6]. Second, the loss of fully $1 / 3$ of all pregnancies, nearly half of them for unknown causes, has a significant impact on the Total Fertility Rate (TFR) of the nation. Although the U.S. TFR has been consistently below replacement level since 1971, virtually nothing is known of the causes for the vast majority of early fetal losses.

The CDC has collected Fetal Death statistics since 1939 when the first Fetal Death Certificate was promulgated. The Cause of Death has been included on this Certificate from the outset. However, the definition of Fetal Death is left to the states, although the CDC does post a recommended definition in the Model State Vital Statistics Act and Regulations (latest revision 1992).

"Each fetal death of 350 grams or more, or if weight is unknown, of 20 completed weeks gestation or more, calculated from the date last normal menstrual period began to the date of delivery, which occurs in this State shall be reported within 5 days after delivery to the (Office of Vital Statistics) or as otherwise directed by the State Registrar. All induced terminations of pregnancy shall be reported in the manner prescribed in Section 16 and shall not be reported as fetal deaths." [7].

As a consequence of this very narrow definition and the voluntary nature of reporting, only about 25,000 fetal deaths are actually reported to the CDC each year, or less than $2.5 \%$ of an estimated 1.1 million natural fetal losses. Since induced abortions are explicitly excluded from the Fetal Death reporting requirements, and there is no mechanism in place to report other early pregnancy losses, matching Live Birth and Fetal Death certificates to women's death certificates overlooks nearly $1 / 3$ of all pregnant women who are at the highest risk for pregnancy-related mortality 
Moreover, if we disaggregate the pregnancies into racial/ethnic groupings (Table 1), we note significant differences among these sub-populations by the proportional composition of pregnancy outcomes. Most notably, less than half of all NHB pregnancies result in a live birth compared to nearly $70 \%$ of non-Hispanic White (NHW) and Hispanic pregnancies. The difference is mostly attributable to an abortions- to - pregnancy rate which is nearly three times higher for NHBs than NHWs. Also of note is that the percent of natural fetal losses-to-pregnancies is higher for NHWs than for Hispanic and NHB pregnancies. Outcome composition of pregnancies for racial/ethnic sub-populations in the U.S., therefore, demonstrates significant differences. Further, these differences are likely to be present in other variously defined sub-populations of comparative research and public policy interest such as individual states or insurance status beneficiary groups.

Table 1. U.S. Pregnancy Outcomes (2009) n, (\%)

\begin{tabular}{|c|c|c|c|c|}
\hline & Pregnancies & Births & Abortions & Fetal Losses \\
\hline ALL & 6369000 & $4131000(64.8)$ & $1152000(18.1)$ & $1087000(17.1)$ \\
\hline HISP & 1474000 & $1000000(67.8)$ & $252000(17.1)$ & $222000(15.1)$ \\
\hline NHB & 1253000 & $615000(49.1)$ & $445000(35.5)$ & $192000(15.3)$ \\
\hline NHW & 3207000 & $222000(69.6)$ & $383000(11.9)$ & $591000(18.4)$ \\
\hline OTHER & 435000 & $281000(64.6)$ & $72000(16.5)$ & $820009(18.9)$ \\
\hline
\end{tabular}

For the same calendar year, 2009, a total of 960 maternal deaths (ICD-10-CM, A34, O00-O99) were reported. If we allocate all these deaths to the appropriate racial/ethnic sub-populations, we can compare maternal mortality ratios using two different denominators: live births and all pregnancies (Table 2).

Table 2. U.S. Maternal Deaths (2009) and Maternal Mortality (MM) Ratios

\begin{tabular}{|c|c|c|c|c|}
\hline & Deaths, $\mathbf{n}$ & $\begin{array}{c}\text { MM Ratio } \\
\text { (births) }\end{array}$ & $\begin{array}{c}\text { MM Ratio } \\
\text { (pregnancies) }\end{array}$ & \% $\boldsymbol{\Delta}$ \\
\hline ALL & 960 & 23.2 & 15.1 & 34.9 \\
\hline HISP & 180 & 18.0 & 12.2 & 32.2 \\
\hline NHB & 305 & 49.6 & 24.3 & 51.0 \\
\hline NHW & 425 & 19.0 & 13.2 & 30.5 \\
\hline OTHER & 50 & 17.8 & 11.5 & 35.3 \\
\hline
\end{tabular}


The overall maternal mortality ratio (MMR) with live births as the denominator is 23.2 deaths per 100,000 live births. At the racial/ethnic sub-population level, we see a NHB MMR which is 2.6 times that of the NHW MMR. If we calculate a MMR using all pregnancies as the denominator, we see a reduction in the overall MMR to 15.1 because the denominators have increased with the inclusion of abortions and fetal losses without an increase in the numerators. Note, however, that MMR reductions are not equal among ethnic groups since the outcome composition differs by racial/ethnic sub-population. With pregnancies as the denominator, the NHB MMR is now only 1.8 times that of the NHW MMR. Therefore, we can see that MMRs are proportionately, but not uniformly, affected by an expansion of the denominator-a function of basic mathematics. However, both of these MMR calculations are based upon an assumption that is known to be false: that all pregnancy outcomes have exactly the same MMR. The research literature clearly indicates that there are significant differences in death rates following specific pregnancy outcomes. Studies from nations with comprehensive reporting of abortion morbidity and mortality, based upon comparison of death certificates of reproductive age woman with all pregnancy related services rendered via a single payer system, and not constrained by a 365 day post termination time frame, conclude that induced abortion or natural fetal loss significantly increases the likelihood of womens' mortality [8-10]. Therefore, meaningful maternal mortality reporting requires not only that all pregnancies be included, but that the overall metric should be disaggregated, numerators and denominators, by the specific pregnancy outcome categories.

When maternal mortality ratios are calculated for each outcome defined sub-population (births, abortions and fetal losses), the results can vary widely, even though the aggregate results will produce the same MMR for all pregnancies irrespective of outcome. Table 3 presents three scenarios in which the 425 NHW maternal deaths in 2009, might be allocated among the three pregnancy outcomes and the resultant changes in outcome-specific MMRs.

Table 3. Three Alternate Scenarios: Outcome Specific Death Counts and Rates, n (\%)

\begin{tabular}{|c|c|c|c|c|}
\hline & $\begin{array}{c}\text { Total } \\
\text { Pregnancies }\end{array}$ & Births & Abortions & Fetal Losses \\
\hline$\# 1$ & 3207000 & $2232000(69.5)$ & $383000(11.0)$ & $591000(18.4)$ \\
\hline & Deaths & 296 & 51 & 78 \\
\hline & MMR & 13.2 & 13.2 & 13.2 \\
\hline & & & & $(20)$ \\
\hline$\# 2$ & Projected percent & $(50)$ & 127 & 85 \\
\hline & Projected Deaths & 213 & 33.1 & 14.4 \\
\hline & Projected MMR & 9.5 & & \\
\hline & & & & \\
\hline
\end{tabular}




\begin{tabular}{|l|l|l|l|l|}
\hline$\# 3$ & Projected percent & $(30)$ & $(40)$ & $(30)$ \\
\hline & Projected Deaths & 127 & 171 & 127 \\
\hline
\end{tabular}

Scenario number one distributes the 425 deaths based on the percent each outcome contributes to total pregnancies. The resultant death allocations are: 296 from births, 51 from abortions and 78 for natural fetal losses. With this allocation the resultant MMRs, calculated with outcome-specific denominators, are exactly the same for each pregnancy outcome, 13.2 deaths per 100,000 births, abortions, or fetal losses. This is the flawed assumption which undermines the interpretation of all maternal mortality statistics. That is, each outcome specific MMR is exactly the same for each defined sub-population. Scenarios 2 and 3 demonstrate how changes in the proportional allocation of deaths among outcomes will change the outcome specific MMRs. As the proportion of fetal losses, for example, increases from $18.4 \%$ to $30 \%$ of all pregnancies, the number of deaths attributed to fetal losses increases from 78 to 127, and the outcome specific MMR increases from 13.2 to 21.5. Similar changes are estimated for births and abortions. Note that in all three scenarios the total of NHW maternal deaths remains 425 and the overall (all outcome) MMR remains 13.2.

\section{Conclusions}

The current measuring metric and reporting methods for calculating maternal mortality exhibit serious inconsistencies, incomplete reporting, incomplete and inaccurate death certificate completion, absence of comprehensive reporting of abortions and natural fetal losses, limitations in the ability to link a maternal death to the appropriate outcome of the pregnancy, and serious limitations and exclusions in the ICD-CM-10 coding system.

Improvement in maternal mortality surveillance in the U.S. and the effectiveness of preventive strategies will benefit from the following actions:

1) Require mandatory certification and reporting of all fetal losses including all categories encompassed by the CDC maternal mortality reporting system: live birth, stillbirth, abortion (disaggregating spontaneous and induced) ectopic pregnancy, and molar pregnancy.

2) Require the department of health of each state to link death certificates, birth certificates, and fetal loss certificates to publish a report each year tabulating the total number of deaths within 42 days, within one year, and one through five years of the decedents' most recent pregnancy outcomes.

3) Modify the structure of the overall maternal mortality ratio metric by changing the denominator from live births to all pregnancies; and enabling specific outcome maternal mortality ratios for live births, stillbirths, spontaneous abortions, induced abortion, ectopic pregnancies, molar pregnancies, and unknown outcomes of pregnancy.

4) Educate providers on where and how to report complications of early pregnancy losses, and work with ICD-10 coding specialists to create more specific abortion complication related codes, with special attention to distinguishing and separating medical from surgical abortion complications. 


\section{Declarations}

Ethics approval and consent to participate: Not Applicable

Consent for publication: Not Applicable

Availability of data and materials: Datasets analyzed in this study are publicly available and the source appropriately referenced in the manuscript

\section{Competing interests:}

The authors declare that they have no competing interests

\section{Funding:}

Not Applicable

\section{Authors' contributions:}

JS: conception and design; analysis and interpretation; draft, revise and approve final manuscript; accountable for all aspects of the work

DCR: interpretation; revise and approve final manuscript

DJH: interpretation; revise and approve final manuscript

JWF: acquisition of data; analysis and interpretation; draft, revise and approve final manuscript

IS: interpretation; revise and approve final manuscript

\section{Acknowledgements:}

Not Applicable

\section{REFERENCES}

1. World Health Organization. (2004) ICD-10: International Statistical Classification Disease and Related Health Problems: Tenth Revision. WHO, Geneva.

2. Hoyert DL. National Center for Health Statistics (US-NCHS-CDC) (2007). Maternal Mortality and Related Concepts. Department of Health and Human Services, Centers for Disease Control and Prevention, National Center for Health Statistics, Hyattsville.

3. Schaible B. 2014. Improving the accuracy of maternal mortality and pregnancy related death. Issues Law Med. 29(2), 231-42. PubMed

4. Curtin SC, Abma JC, Ventura SC. NCHS Data Brief No. 136, December 2013. Centers for Disease Control and Prevention, National Center for Health Statistics, Atlanta. 
5. Gissler M, Berg C, Bouvier-Colle M-H, Buekens P. 2005. Injury deaths, suicides and homicides associated with pregnancy, Finland 1987-2000. Eur J Public Health. 15(5), 45963. doi:https://doi.org/10.1093/eurpub/cki042. PubMed

6. Reardon DC, Ney PG, Scheuren F, Cougle J, Coleman PK, et al. 2002. Deaths associated with pregnancy outcome: a record linkage study of low income women. South Med J. 95(8), 83441. PubMed https://doi.org/10.1097/00007611-200295080-00011

7. Model State Vital Statistics Act and Regulations, 1992 Revision, February 1994. Centers for Disease Control and Prevention/National Center for Health Statistics (CDC/NCHS).

8. Reardon DC, Thorp JM. 2017. Pregnancy associated death in record linkage studies relative to delivery, termination of pregnancy, and natural losses: A systematic review with a narrative synthesis and meta-analysis. SAGE Open Med. 5, 1-17. doi:https://doi.org/10.1177/2050312117740490. PubMed

9. Reardon DC, Coleman PK. 2012. Short and long term mortality rates associated with first pregnancy outcome: Population register based study for Denmark 1980-2004. Med Sci Monit. 18(9), PH71-76. doi:https://doi.org/10.12659/MSM.883338. PubMed

10. Gissler M, Kauppila R. 1997. Merilainen d. Pregnancy associated deaths in Finland 19871994: Definition problems and benefits of record linkage. Acta Obstet Gynecol Scand. 76, 651-57. PubMed https://doi.org/10.3109/00016349709024605 